\title{
The metabolic activity of neutrophils measured with a chemiluminescence assay in young patients with type 1 diabetes depending on the disease duration
}

\author{
MAGDALENA KACZMAREK ${ }^{I^{*}}$, ZDZISŁAWA IWANICKA ${ }^{2 * *}$, ADAM JANKOWSKI ${ }^{1,2}$, \\ ALEKSANDRA LEWANDOWICZ-USZYNSSKA ${ }^{3}$,
}

${ }^{1}$ Institute of Genetics and Microbiology, University of Wroclaw, Poland

${ }_{1}$ Student Scientific Society at the $3^{\text {rd }}$ Department and Clinic of Paediatrics, Immunology and Rheumatology of Developmental Age, Wroclaw Medical University, Poland

2Department and Clinic of Endocrinology, Wroclaw Medical University, Poland Current place of employment:

33rd Department and Clinic of Paediatrics, Immunology and Rheumatology of Developmental Age, Wroclaw Medical University, Poland

*Rex Company, National Genetics Centre, Wrocław

**Provincial Health Care Specialist Group in Wrocław

\begin{abstract}
The study deals with the estimation of the metabolic activity of neutrophils measured with a chemiluminescence assay in children with type I diabetes depending on the disease duration, compared to the control group. The study involved 87 children with type I diabetes aged 4 to 21 years (52 girls, 35 boys). Patients were divided into four subgroups depending on the disease duration: subgroup I- the disease duration up to 12 months (35 children), subgroup II - the disease duration up to 5 years (28 children), subgroup III - the disease duration up to 10 years (12 children), and subgroup IV - the disease duration over 10 years (12 children). The control group was composed of 23 persons - healthy volunteers aged 20 to 25 years (mean age 23 years). The metabolic activity of neutrophils was determined in a luminol-dependent chemiluminescence assay. The spontaneous and stimulated chemiluminescence of neutrophils (fMLP, opsonised Zymosan, PMA) was tested on a 96-well microplate (LB96P-WMP). The chemiluminescence assay was conducted using a luminometer (MicroLumat LB 96P of E\&G $B A R T H O L D)$. The results have been written in RLU units (Relative Light Units/s) as the maximum value of chemiluminescence (CLmax). The test showed statistically significantly lower values of spontaneous and stimulated neutrophil chemiluminescence in all subgroups, compared to the control group. The lowest levels of neutrophil CL were in subgroup III (neutrophils stimulated with FMLP and PMA) and also in children with newly detected diabetes (Zymosan-stimulated neutrophils).
\end{abstract}

Key words: diabetes, children, neutrophil chemiluminescence, disease duration.

(Centr Eur J Immunol 2013; 38 (2): 237-242)

\section{Introduction}

Many pathologies result from physical and chemical reactions that lead to the formation of highly reactive forms of oxygen referred to as free radicals $[1,2]$. They originate mainly from processes occurring inside granulocytes which activate the NADPH oxidase enzyme system, which produces a phenomenon called a respiratory burst [3]. Released RT are necessary for intracellular destruction of many microorgan- isms, acting with granulocyte enzymes as an essential part of the immune system that protects against most pathogens. RT released into the extracellular space or as a result of excessive activation of neutrophils, or due to a decreased activity of enzymes neutralizing their activity (SOD, catalase, peroxidase), or as a result of lipid peroxidation, lead to the formation of protein oxidation products, and damage the surrounding cells and even tissues, leading to the development

Correspondence: dr n. med. Aleksandra Lewandowicz-Uszyńska, $3^{\text {rd }}$ Department of Paediatrics, Clinic of Immunology and Rheumatology of Developmental Age, Wroclaw Medical University, ul. Koszarowa 5, 51-149 Wrocław, Poland, tel. +48 713925 397, fax +48 713925 396, e-mail: alusz1 @ poczta.onet.pl 
of inflammation and autoimmune processes [4-7]. Diabetes is one of the conditions in which the processes leading to the damage of pancreatic islet $\beta$ cells are closely related to the free radical pathology [8-11]. Elevated blood glucose increases oxidative stress and release of free radicals, thereby causing the activation of NF- $\kappa \mathrm{B}$ [12]. It is believed that excessive activation of neutrophils may occur a few or more months ahead of the onset of symptoms, and the then produced RT along with a network of pro-inflammatory cytokines, nitric oxide, and autoreactive T cells may in favourable conditions (genetic background, infections, stress, dietary errors) lead to the destruction of the islets of Langerhans [13-17].

Therefore, the aim of this study was to assess the metabolic activity of neutrophils in children with type 1 diabetes, depending on the disease duration, compared to the control group.

\section{Material and methods}

The study involved 87 children with type 1 diabetes aged 4 to 21 years ( 52 girls and 35 boys, mean age 13 years) who were patients of the Clinic of Developmental Endocrinology, Wroclaw Medical University (in 2000/2001). Depending on the disease duration, patients were divided into four subgroups:

- subgroup I - the disease duration up to 12 months (35 children),

- subgroup II - the disease duration up to 5 years (28 children),

- subgroup III - the disease duration up to 10 years (12 children),

- subgroup IV - the disease duration over 10 years (12 children).

The control group consisted of 23 people - healthy volunteers aged 20 to 25 years (mean age 23 years).

All patients were receiving humanized insulin injections several times a day from the early onset of diabetes mellitus. The study was approved by the Bioethics Committee (858/0103) at the Wroclaw Medical University.

The material for this study was venous blood collected during basic diagnostic tests or checks into plastic tubes with heparin in a closed system. The metabolic activity of neutrophils was measured using a luminol-dependent chemiluminescence assay. The spontaneous and stimulated neutrophil CL was studied (fMLP, opsonised Zymosan, PMA) in a 96-well white microplate (LB96P-WMP) according to Lewkowicz et al. [18]. The order of addition and volume of reagents are shown below:

$\begin{array}{ll}\text { No stimulation: } & \text { FMLP: } \\ \text { 1. PBS } 40 \mu \mathrm{l} & \text { 1. PBS } 20 \mu 1 \\ \text { 2. Luminol } 20 \mu 1 & \text { 2. fMLP } 20 \mu 1 \\ \text { 3. Whole blood } 40 \mu 1 & \text { 3. Luminol } 20 \mu 1 \\ & \text { 4. Whole blood } 40 \mu 1\end{array}$

PMA:

1. PBS $20 \mu 1$

2. PMA $20 \mu 1$

3. Luminol $20 \mu \mathrm{l}$

4. Whole blood $40 \mu \mathrm{l}$
Zymosan:

1. PBS $20 \mu 1$

2. Zymosan $20 \mu 1$

3. Luminol $20 \mu 1$

4. Whole blood $40 \mu 1$
Each of the systems was repeated three times, and the arithmetic mean of the values received was calculated. The study was conducted using a chemiluminescence luminometer (LB 96P MicroLumat of E\&G Barthold). The device was controlled by WinGlow software in the Windows environment. Spot measurements were taken within 0.2 seconds every 60 seconds at $37^{\circ} \mathrm{C}$. The test took 45 minutes. Results are expressed in RLU (Relative Light Units/s) as the maximum chemiluminescence $\left(\mathrm{CL}_{\max }\right)$. Since $\mathrm{CL}$ depends linearly on the number of neutrophils, while heme compounds reduce its value, the chemiluminescence results were adjusted to their absolute number and haemoglobin content in the blood tested by the formula:

$$
\mathrm{CL}_{\text {CALCULATED }}=\mathrm{CL}_{\text {DETERMINED }} \times \frac{\mathrm{Hb}[\%]}{\mathrm{PMN}[\%] \times \mathrm{WBC}[\text { thous. } / 100 \mu \mathrm{l}]}
$$

where $\mathrm{Hb}$ is haemoglobin and PMN is the percentage of neutrophils in the total number of white blood cells (WBC).

A statistical analysis conducted using the Mann-Whitney $U$ test involved determination of the arithmetic mean, standard deviation, and the level of significance $(p<0.05)$ for each parameter of neutrophil CL in examined patients and the control group. Using the Kruskal-Wallis ANOVA by Ranks test, box and whisker plots were made showing individual parameters of neutrophil CL including the mean, standard deviation and standard error for examined patients and the control group.

\section{Results}

The results of spontaneous neutrophil CL are summarized in Fig. 1. Significantly lower CL values were shown in all children with diabetes, regardless of the disease duration. There were no statistically significant differences in spontaneous neutrophil CL values between subgroups I and II, II and III, III and IV, I and III, I and IV. Significantly lower values of spontaneous CL were observed in subgroup IV in comparison with the results obtained in subgroup II. The effect of fMLP stimulation of neutrophils in the examined group is presented in Fig. 2. Significantly lower values of neutrophil CL were demonstrated in subgroups I, III and IV, compared to the control group. There were no statistically significant differences between the $\mathrm{CL}_{\max }$ levels in subgroup II, compared to healthy subjects. The lowest values of CL after stimulation with fMLP were found in subgroup III, i.e. in children with diabetes in the time interval from 5 to 10 years. There were no statistically signifi- 


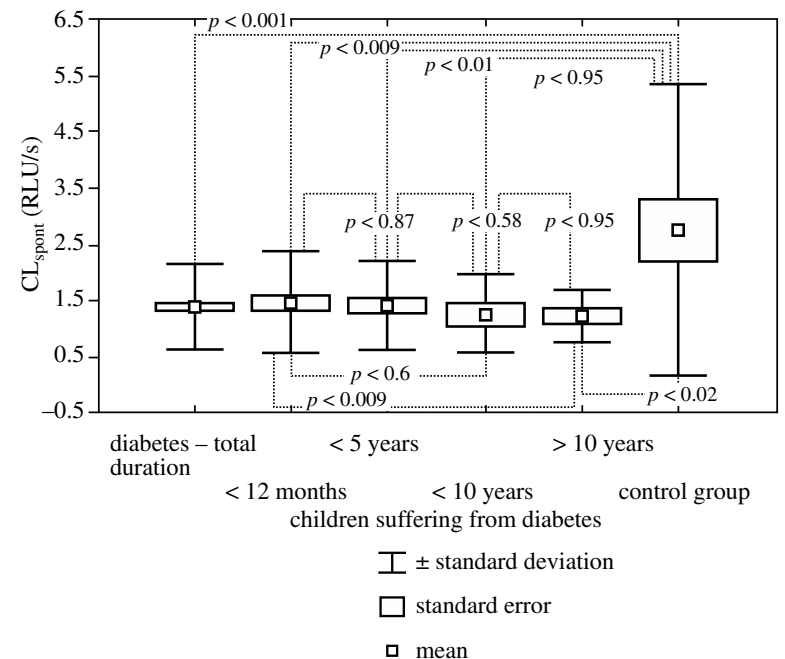

Fig. 1. Spontaneous chemiluminescence of neutrophils in children with diabetes compared to the control group

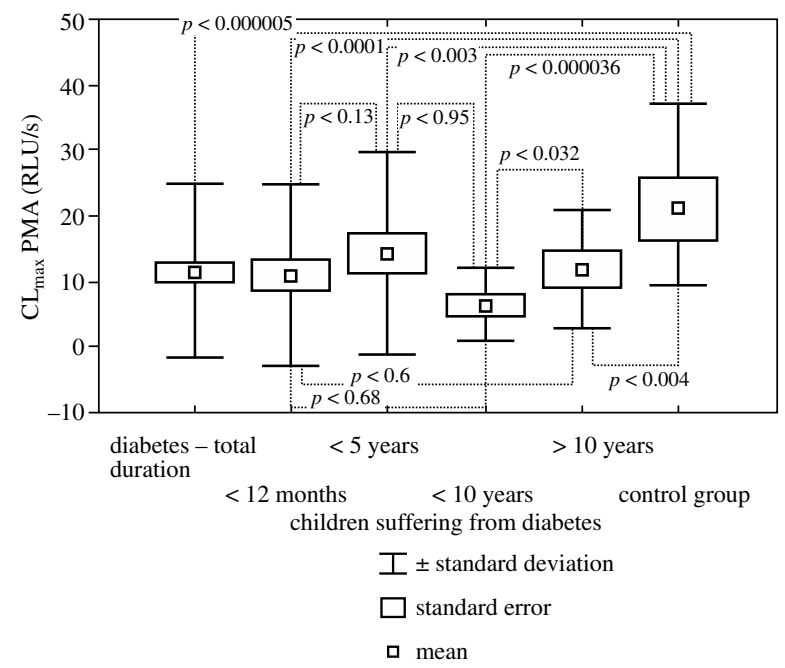

Fig. 3. The maximum value of neutrophil chemiluminescence after PMA stimulation in children compared to the control group

cant differences in $\mathrm{CL}_{\max }$ levels between subgroups I and II, II and III, III and IV, and between I and III, I and IV, II and IV. Neutrophil response to stimulation with PMA is given in Fig. 3. All subgroups of children with diabetes had significantly lower $\mathrm{CL}_{\max }$ values, compared to the control group. The weakest response to PMA stimulation was observed in subgroup III (i.e. in children in whom the disease lasts from 5 to 10 years). There were no statistically significant differences in the maximum values of neutrophil CL between subgroups I and II, I and IV, and II

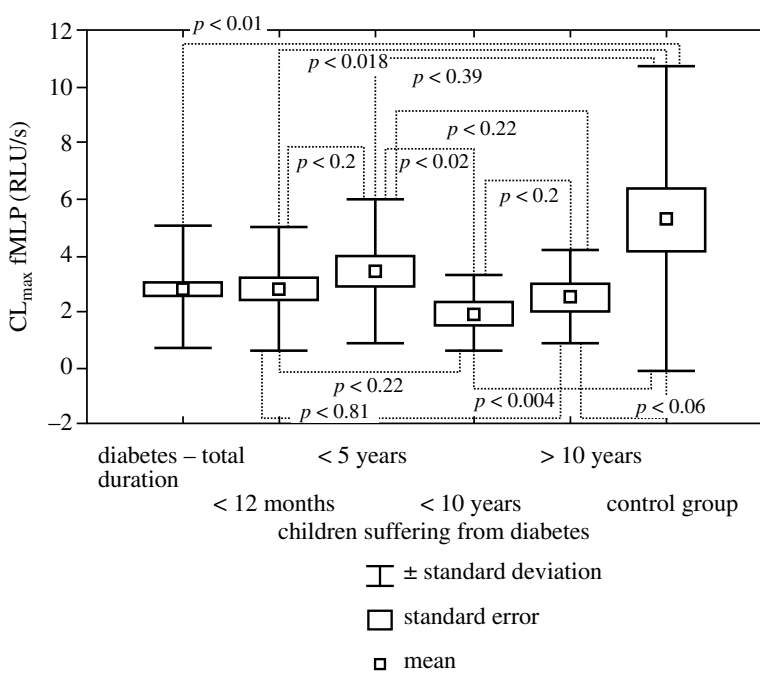

Fig. 2. The maximum value of neutrophil chemiluminescence after fMLP stimulation in children compared to the control group

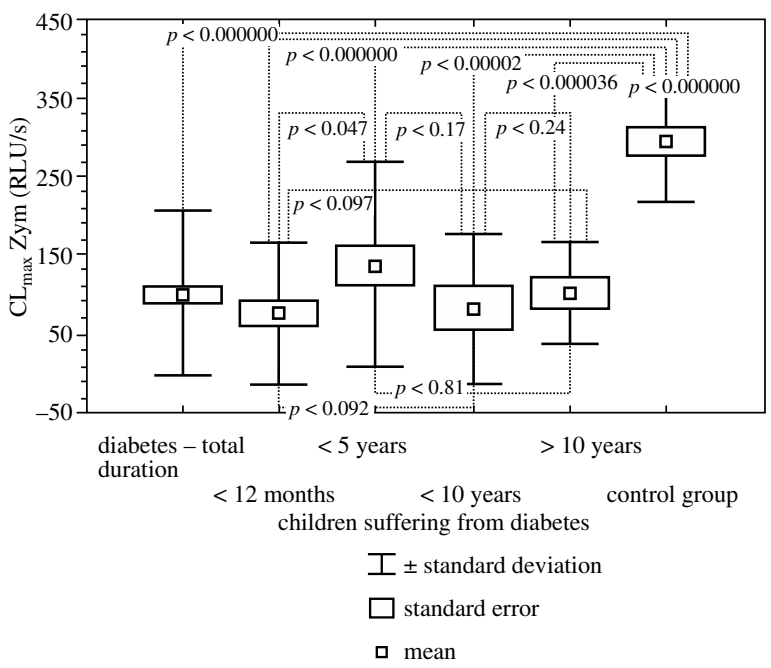

Fig. 4. The maximum value of neutrophil chemiluminescence after opsonised Zymosan stimulation in children diabetes compared to the control group

and IV. Figure 4 presents $\mathrm{CL}_{\max }$ results after stimulation with opsonised Zymosan. Significantly lower neutrophil CL was reported in all the examined subgroups, compared to the control group. The lowest levels of neutrophil CL were observed in children with newly detected diabetes. There were statistically significant differences in $\mathrm{CL}_{\max }$ between subgroups I and II, and I and IV. There were no statistically significant differences in the values of Zymosan-activated neutrophil CL between subgroups II and III, III and IV, as well as I and III, II and IV. 


\section{Discussion}

Maintaining the stability of the neutrophils-RTantioxidant enzymes system determines maintaining RT concentrations at the appropriate level that is sufficient to protect against infectious agents, without causing damage associated with excessive proliferation of these compounds. Telci et al. [19] showed elevated levels of markers of oxidative damage to proteins and decreased plasma antioxidant capacity in children with type 1 diabetes, compared to the control group. Studies showing impaired neutrophil oxidative metabolism in patients with diabetes were conducted as early as in the 1990s [20, 21]. Decreased oxidative metabolism of neutrophils measured with a chemiluminescence assay was also demonstrated in children with type 1 diabetes in a variety of clinical conditions (good, medium, severe), compared to healthy subjects [22]. In the present study, all tested systems (spontaneous and stimulated CL) showed impaired neutrophil oxidative metabolism in all subgroups of children with diabetes, compared with the control group. Thus, impaired response of neutrophils to both receptor stimulation (fMLP, Zymosan) and non-receptor stimulation (PMA) was reported. A similar observation was made by Nishizawa et al. [23] who showed impaired neutrophil CL after stimulation with fMLP in patients with type 1 diabetes, compared to healthy subjects. Kantara et al. [24] reported significantly enhanced spontaneous CL in patients with diabetes compared to healthy subjects, which was not observed in the present study. PMA stimulation of neutrophils resulted in convergent observation, i.e. a significant reduction in CL in comparison with healthy subjects. It is worth noting that the lowest CL values were found in children from subgroup III (after stimulation with fMLP and PMA). In subgroup IV, i.e. in children with the longest disease duration (over 10 years) CL results (after stimulation with PMA) were statistically significantly higher than in children from subgroup III. Longer duration of diabetes increases the risk of the so-called late complications (microangiopathy, vascular lesions) directly related to RT activity [25]. Perhaps the slight increase of CL observed in children with the longest disease duration indicates the beginning of the process. It is noteworthy that the lowest CL levels (after stimulation with Zymosan) were reported in children with newly detected diabetes. This may indicate that neutrophil dysfunctions (related to the processes of phagocytosis and CL) occur at an early stage of the disease. Descamps et al. [26] showed no changes in spontaneous neutrophil CL in patients with newly detected diabetes (the tests were performed 2 months after the onset of diabetes). After stimulation with fMLP and PMA, the researchers observed enhanced neutrophil CL. After three months of treatment, CL reached the normal values of healthy subjects. Unfortunately, the research was not continued in a longer time interval. The fact of poor response to this type of neutrophil stimulation explains the tendency of patients with suspected or newly detected diabetes to have frequent infections during this period. In the present study, the situation observed in children with diabetes was similar to that of recurrent respiratory infections in children and adults [27-29], in which a decline in neutrophil CL, probably resulting from recurrent infections, was observed. Perhaps chronic activation leads to the depletion of neutrophil energy reserves and consequently to a decline in their CL. The possibility of the existence of different functional states of neutrophils, including the state of "exhaustion", has been described by various authors [30]. In this study chronic preactivation of neutrophils in a high concentration of glucose in the body can decrease the sensitivity of these cells to subsequent stimuli. The reduced neutrophil CL in children with type 1 diabetes observed in this study is in apparent contradiction to the fact of excessive activity of neutrophils in the pathophysiology of diabetes. It must be noted that the oxidative burst of neutrophils is influenced by various factors, such as certain drugs, impaired glucose profile, insulin, blood ph disorders associated with the accumulation of acidic metabolites, and finally elevated levels of haemoglobin and its derivatives which significantly reduce neutrophil CL in whole blood [31,32]. The presence of elevated levels of glycated haemoglobin $\left(\mathrm{HbA}_{1 \mathrm{C}}\right)$ in erythrocytes in patients with uncontrolled diabetes may be the essence of this phenomenon. Studies conducted in vitro by Oldenborg et al. [31] on healthy people showed inhibition of neutrophil chemiluminescence after stimulation with fMLP by insulin. Gallacher et al. [34] found lower CL values in adults with diabetes, compared to the control group. The authors also found a negative correlation between blood levels of chemiluminescence and $\mathrm{HbA}_{1 \mathrm{C}}$. Thus, the weakening of neutrophil bactericidal function should be associated not only with direct changes in blood glucose levels, but also with poor metabolic control of the disease. Another cause of impaired neutrophil oxidative metabolism may be the response of these cells to insulin. Saeed et al. [27] showed impaired neutrophil oxidative metabolism using a CL array in patients with diabetes, and they attributed the inhibitory role to acidic metabolites (acetoacetate) present in the serum of these patients. Delamaire et al. [36] also showed impaired neutrophil oxidative metabolism (chemotaxis, chemiluminescence) in patients with type 1 diabetes. Just as in the present study, the PMA and Zymosan-stimulated CL of neutrophils was lower, compared to healthy subjects. Marhoffer et al. [37] demonstrated lower neutrophil CL after stimulation with PMA in diabetic patients, compared to healthy individuals. Assessing the phenomena associated with RT generation, we should, if possible, include processes occurring inside neutrophils as well as processes associated with the so-called extracellular CL resulting from lipid peroxidation of cell membranes (directly independent of NADPH oxidase), however, as a consequence leading to tissue damage and the accumulation of metabolites (malonic aldehyde) associated with the activity of other free radicals and nitric oxide [38]. 


\section{Conclusions}

Children with diabetes, regardless of the disease duration, had impaired spontaneous oxidative metabolism of neutrophils measured with a chemiluminescence assay in comparison to the control group.

The authors declare no conflict of interest.

\section{References}

1. Zozulińska D, Wierusz-Wysocka B (2000): Wybrane mechanizmy patogenetyczne przewlekłych powikłań cukrzycy. II Rola granulocytów obojętnochłonnych. Diabetol Pol 7: 198.

2. Valko M, Leibfritz D, Moncol J, et al. (2007): Free radicals and antioxidants in normal physiological functions and human disease. Int J Biochem Cell Biol 39: 44-84.

3. Allen RC (1986): Phagocytic leukocyte oxygenation activities and chemiluminescence: a kinetic approach to analysis. Methods Enzymol 133: 449-493.

4. Pinzani P, Petruzzi E, Orlando C, et al. (1998): Serum antioxidant capacity in healthy and diabetic subjects as determined by enhanced chemiluminescence. J Biolumin Chemilumin 13: 321-325.

5. Gil-del Valle L, de la Milian LC, Toledo A, et al. (2005): Altered redox status in patients with diabetes mellitus type 1. Pharmacol Res 51: 375-380.

6. Kantárová D, Buc M, Stuchlíková M, et al. (2006): Etiopatogensis of autoimmune diabetes mellitus in human. A review. Centr Eur J Immunol 31: 102-110.

7. Salmanowicz B, Noczyńska A, Krzystek-Korpacka M (2009): Evaluation of markers of oxidative proteins (AOPP) and lipids (TBARs) in children with diabetes type 1. Endokrynol Ped 1: 33-44.

8. Arai K, lizuka S, Tada Y, et al. (1987): Increase in the glucosylated form of erythrocyte $\mathrm{Cu}-\mathrm{Zn}$-superoxide dismutase in diabetes and close association of the nonenzymatic glucosylation with the enzyme activity. Biochim Biophys Acta 926: 292-296.

9. Dandona P, Thusu K, Cook S, et al. (1996): Oxidative damage to DNA in diabetes mellitus. Lancet 17: 347: 444-445.

10. Davison GW, George L, Jackson SK, et al. (2002): Exercise, free radicals, and lipid peroxidation in type 1 diabetes mellitus. Free Radic Biol Med 33: 1543-1551.

11. Hopps E, Camera A, Caimi G (2008): Polimorphonuclear leukocytes and diabetes mellitus. Minerva Med 99: 197-202.

12. Evans JL, Goldfine ID, Maddux BA, Grodsky GM (2003): Are oxidative stress-activated signaling pathways mediators of insulin resistance and $\beta$-cell dysfunction? Diabetes 52: 1-8.

13. Kolb H, Kolb-Bachofen V (1992): Type 1 (insulin-dependent) diabetes mellitus and nitric oxide. Diabetologia 35: 796-797.

14. Komosińska K, Olczyk K (1995): The role of free radicals in pathogenesis of insulin dependent diabetes mellitus. Post Hig Med Dośw 49: 733-746.

15. Rösen P, Nawroth PP, King G, et al. (2001): The role of oxidative stress in the onset and progression of diabetes and its complications: a summary of Congress Series sponsored by UNESCO-MCBN, the American Diabetes Association and the German Diabetes Society. Diabetes Metab Res Rev 17: 189-212.
16. Maritim AC, Sanders RA, Watkins JB 3rd (2003): Diabetes, oxidative stress, and antioxidants: a review. J Biochem Mol Toxicol 17: 24-38.

17. Hatanaka E, Monteagudo PT, Marrocos MS, Campa A (2006): Neutrophils and monocytes as potentially important sources of proinflammatory cytokines in diabetes. Clin Exp Immunol 146: 443-447.

18. Lewkowicz P, Lauk-Pachuła B, Górańska N, et al. (1999): The attempt of standardization of the whole blood chemiluminescence assessment as a method of examining the human granulocytes' function in the "in vitro" research. Diagn Lab 35: 497-510.

19. Telci A, Cakatay U, Salman S, et al. (2000): Oxidative protein damage in early stage type 1 diabetic patients. Diabetes Res Clin Pract 50: 213-223.

20. Marhoffer W, Stein M, Schleinkofer L, Federlin K (1994): Monitoring of polymorphonuclear leukocyte functions in diabetes mellitus - a comparative study of conventional radiometric function tests and low-light imaging systems. J Biolumin Chemilumin 9: 165-170.

21. Nerup J, Mandrup-Poulsen T, Helqvist S, et al. (1994): On the pathogenesis of IDDM. Diabetol 37: S82-S89.

22. Kaczmarek M, Lewandowicz-Uszyńska A, Iwanicka Z, et al. (2012): Whole blood neutrophil chemiluminescence with diabetes depending on their clinical condition. Cent Eur J Immunol 37: 345-349.

23. Nishizawa Y, Amakata Y, Fushiki S, et al. (1999): Improvement of an impaired chemiluminescence response to formylmethionyl-leucyl-phenylalanine in neutrophils from patients with non insulin dependent diabetes mellitus by recombinant human granulocyte-colony stimulating factor. In Vivo 13: 319-325.

24. Kantar A, Wilkins G, Swoboda B, et al. (1990): Alterations of the respiratory burst of polymorphonuclear leukocytes from diabetic children. A chemiluminescence study. Acta Paediatr Scand 79: 535-541.

25. Johansen JS, Harris AK, Rychly DJ, Ergul A (2005): Oxidative stress and the use of antioxidants in diabetes: linking basic science to clinical practice. Cardiovasc Diabetol 4: 1-11.

26. Descamps-Latscha B, Nguyen AT, Feutren G (1990): Phagocyte oxidative metabolism in cyclosporine- or placebo-treated patients with insulin-dependent (type I) diabetes mellitus of recent onset. J Autoimmun 3: 201-213.

27. Sikora JP (1996): Chemiluminescent assessment of aerobic metabolism of peripheral blood polymorphonuclear leukocytes in children with increased susceptibility to respiratory tract infections. Centr Eur J Immunol 21: 33-38.

28. Zabuska-Jabłońska K, Broniek A (1997): Disturbances of polymorphonuclear neutrophil leukocyte chemiluminescence in patients with chronic or recurrent respiratory tract infections. Pneumol Allergol Pol 65: 649-659.

29. Lewandowicz-Uszyńska A, Jankowski A (2001): Application of neutrofils chemiluminescence test in the differential diagnosis of asthma and rrti the remission period in children. Proc SPIE 91: 4515-4514.

30. Zgliczyński JM, Kwasnowska E, Stelmaszyńska T, et al. (1988): Functional states of neutrophils as suggested by whole blood chemiluminescence. Acta Biochim Polon 35: 331-342.

31. Hunt JV, Dean RT, Wolff SP (1988): Hydroksyl radical production and autoxidative glycosylation. Glucose autooxide as the cause of protein damage in the experimental glycation model of diabetes mellitus and ageing. Biochem J 256: 205-212. 
32. Scatena R, Nocca G, De Sole P, et al. (1998): Impaired reactive oxygen metabolism of phagocytic leukocytes in NIDDM patients. A role for non-enzymatic glycosylation of collagen. J Biolumin Chemilumin 13: 273-278.

33. Oldenborg PA (1999): Effects of insulin on N-formylmethionyl-leucyl-phenylalanine (fMet-Leu-Phe)-stimulated production of reactive oxygen metabolites from normal human neutrophils. Inflamm Res 48: 404-411.

34. Gallacher SJ, Thomson G, Fraser WD, et al. (1995): Neutrophils bactericidal function in diabetes mellitus: evidence for association with blood glucose control. Diabet Med 12: 916-920.

35. Saeed FA, Castle GE (1998): Neutrophil chemiluminescence during phagocytosis is inhibited by abnormally elevated levels of acetoacetate: implications for diabetic susceptibility to infections. Clin Diagn Lab Immunol 5: 740-743.

36. Delamaire M, Maugendre D, Moreno M, et al. (1997): Impaired leucocyte functions in diabetic patients. Diabet Med 14: 29-34.

37. Marhoffer W, Stein M, Schleinkofer L, Federlin K (1993): Evidence of ex vivo and in vitro impaired neutrophil oxidative burst and phagocytic capacity in type 1 diabetes mellitus. Diabetes Res Clin Pract 19: 183-188.

38. Erciyas F, Taneli F, Arslan B, Uslu Y (2004): Glycemic control, oxidative stress, and lipid profile in children with type 1 diabetes mellitus. Arch Med Res 35: 134-140. 